\title{
Fingerprint IR Spectroscopy to Probe Amino Acid Conformations in the Gas Phase
}

\author{
Joost M. Bakker, ${ }^{1, *}$ Luke Mac Aleese, ${ }^{1,2}$ Gerard Meijer, ${ }^{1,3,4}$ and Gert von Helden ${ }^{1}$ \\ ${ }^{1}$ FOM-Institute for Plasmaphysics Rijnhuizen, Edisonbaan 14, NL-3439 MN Nieuwegein, The Netherlands \\ ${ }^{2}$ Laboratoire de Chimie Physique (UMR 8000-CNRS), Bâtiment 350, Université Paris XI, 91405 Orsay CEDEX, France \\ ${ }^{3}$ Department of Molecular and Laser Physics, University of Nijmegen, Toernooiveld, NL-6525 ED Nijmegen, The Netherlands \\ ${ }^{4}$ Fritz-Haber-Institut der Max-Planck-Gesellschaft, Faradayweg 4-6, D-14195 Berlin, Germany
}

(Received 25 June 2003; published 12 November 2003)

\begin{abstract}
We report the infrared (IR) absorption spectra of different conformational isomers of gas phase amino acid molecules in the molecular fingerprint region of 330-1500 $\mathrm{cm}^{-1}$. The IR absorption spectra for three conformers of the amino acid tryptophan show absorption bands that uniquely identify the conformational structure of the molecule and that are well matched by density functional theory calculations. The present observations hold great promise for future identification of conformational folding of larger molecules by means of their IR absorption characteristics.
\end{abstract}

Over the past 20 years, sophisticated techniques have been developed to bring intact large biomolecules into the gas phase. The techniques most widely used today are matrix-assisted laser desorption ionization (MALDI) [1] and electrospray ionization (ESI) [2]. Because of their ability to provide mass and sequence information of biological samples, both techniques have revolutionized analytical biochemistry. However, inferring structural information on gas phase ions and molecules is often a difficult task. The knowledge of the gas phase structure can provide important insight into fundamental intramolecular interactions and can serve as a calibration point for theoretical models. While elaborate tools for solid and liquid samples exist, most of them cannot be applied to gas phase samples. One of the few techniques that can provide direct structural information on gas phase species is ion mobility, in which large ionized molecules are mass selected and pass through a drift cell filled with a buffer gas $[3,4]$. The molecular ions will arrive at the end of the drift cell after a period of time which depends on their collisional cross section. Information can also be obtained using spectroscopic methods, either in the ultraviolet (UV) [5] or in the infrared (IR) [6,7]. The IR absorption spectrum is a unique identifier for the structure: line intensities and frequencies give direct information on the forces that hold the molecule together. Recently, it was experimentally demonstrated that additional information can be obtained by the direct measurement of the direction of the vibrational transition dipole moment of isolated species [8]. Selective IR induced isomerization in the gas phase is another interesting technique that provides structural and dynamical information [9]. We here present IR absorption spectra for three conformers of tryptophan in the gas phase over a wide frequency range, from 330 to $1500 \mathrm{~cm}^{-1}$. It is observed that the conformation has a strong influence on the spectra. The experimental results are combined with density functional theory (DFT) cal- culations, and unique information on the structures of the individual conformers is obtained.

IR absorption spectra of gas phase species can be obtained by ion- or fluorescence-dip spectroscopy $[6,7,10]$, a technique that has been applied to various interesting systems, such as isolated [11] or paired nucleobases [12], to the amino acids phenylalanine and tryptophan $[13,14]$, and to $\beta$-sheet model systems $[15,16]$. These experiments have all been performed using laser systems that cover the near- and mid-IR and are limited to wavelengths shorter than about $7 \mu \mathrm{m}$. In this range, $X-\mathrm{H}(X=\mathrm{C}, \mathrm{O}, \mathrm{N})$, and $\mathrm{C}=\mathrm{O}$ stretching vibrations are probed and the experiments provide insight into the conformational arrangement, as transitions exhibit shifts in absorption frequency and changes in intensity in the presence of, for instance, intramolecular hydrogen bonds or solvating molecules. However, most vibrational modes have characteristic frequencies that lie further in the IR and are not accessible using standard laser systems.

The amino acid tryptophan has been studied extensively by various groups as it absorbs strongly in the near$\mathrm{UV}$ and as it is an important probe in fluorescence spectroscopy [17]. Even though tryptophan is a relatively simple molecule, in the gas phase it is observed to fold into several different conformers. The various conformers have different electronic transitions and can be selectively excited using UV lasers [18]. A detailed structural assignment, however, requires more information. IR spectra in the $X-\mathrm{H}$ spectral region have recently been obtained for different conformers and, combined with DFT, a first assignment to specific geometries is obtained [14].

The present IR absorption spectra of tryptophan conformers in the region of $330-1500 \mathrm{~cm}^{-1}$ are measured in a pulsed molecular beam experiment with mass and conformer specific detection. Gas phase tryptophan molecules are evaporated and entrained by an argon gas pulse of typically $200 \mu$ s duration. In the following 
supersonic expansion into vacuum, molecules are internally cooled. After passing through a skimmer, they interact with pulsed UV and IR laser beams at the crossing point of the mutually perpendicular molecular beam axis, laser beam axis, and the flight tube axis of a timeof-flight mass spectrometer in which UV laser produced ions are detected.

The IR absorption spectra are measured making use of IR ion-dip spectroscopy (IR-IDS), which is schematically depicted in Fig. 1. In this technique, the internally cooled molecules are resonantly ionized by a pulsed UV laser that is tuned to the transition frequency of one selected conformer, to produce a constant ion signal. A few $\mu$ s before the UV laser fires, the IR laser interacts with the molecules. If a vibrational transition in the molecules is induced by the IR light, population is transferred into an excited vibrational state, yielding a depletion of ground state molecules. This results in a reduction of the number of ions produced. By measuring the ion signal while tuning the IR wavelength, an ion-dip spectrum is recorded. As a widely tunable source of IR radiation, a free-electron laser (FEL) is ideal for these studies [19]. The experiments presented here make use of "FELIX," the free-electron laser for infrared experiments [20,21]. The FELIX output consists of a few- $\mu$ s long burst (macropulse) of micropulses. The micropulse spacing within the burst is set to $1 \mathrm{~ns}$, while the macro-

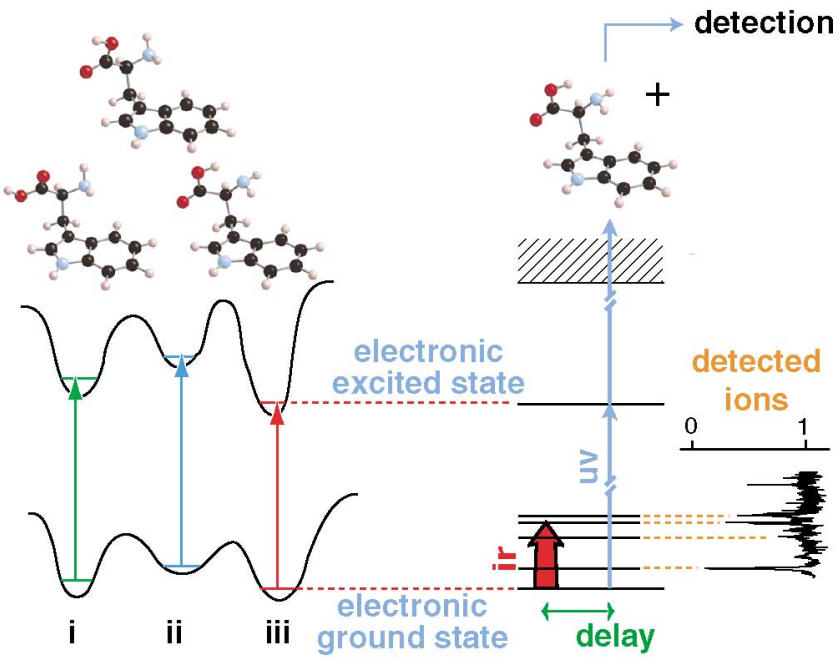

FIG. 1 (color). Scheme of the infrared ion-dip spectroscopy (IR-IDS) experiment. The figure shows several conformers of tryptophan as different minima on the potential energy surfaces. As the relative energies of the different conformers in the ground and electronically excited state will differ, the excitation energies to the electronically excited state will differ as well. As a consequence, a single conformer can be selectively excited and ionized by a UV laser. An IR laser, fired prior to the UV laser, can then transfer population out of the ground state into a vibrationally excited state, resulting in a dip in the ion signal. pulses are repeated at $10 \mathrm{~Hz}$. The micropulse duration is set to about 100 optical cycles, which results in a bandwidth of approximately $1 \%$ (FWHM) of the central frequency. The wavelength range that can be covered extends from 40 to $2000 \mathrm{~cm}^{-1}$ although, in this experiment, only the region between 300 and $1500 \mathrm{~cm}^{-1}$ is used. In a macropulse, typically, energies of up to $100 \mathrm{~mJ}$ can be reached.

In Fig. 2, the IR absorption spectra in the range between 330 and $1500 \mathrm{~cm}^{-1}$ are displayed for three conformers, labeled $\mathrm{A}, \mathrm{D}$, and $\mathrm{E}$, where the notation introduced by Rizzo et al. is used [18]. The relative IR absorption cross sections are obtained as the natural logarithm of the IR laser induced ion-dip signal, normalized to the constant ion current produced by the UV laser. The absolute frequency accuracy is about $3 \mathrm{~cm}^{-1}$ at the low frequency end of the spectrum, gradually deteriorating to $15 \mathrm{~cm}^{-1}$ at the high frequency end. The linewidth observed in the spectrum is almost exclusively determined by the bandwidth of the laser, and is about $1 \%$ of the FELIX frequency.

One can immediately observe that the three spectra are different and that the conformation has a large effect on the vibrational structure. The strong resonance near $1400 \mathrm{~cm}^{-1}$ in conformer A, for instance, is not present in the spectra of the other two conformers. The region below $700 \mathrm{~cm}^{-1}$ appears to show the most pronounced differences. As this is the region where vibrations that involve floppy, large-amplitude motions are expected, this low frequency range should be especially sensitive to the conformation of the molecule.

To analyze the observed spectra, DFT calculations are performed to find the most stable geometries for tryptophan. A total of 32 starting geometries are submitted to a full geometry optimization using the B3LYP functional with the $6-31+G(d)$ basis set. The starting geometries are obtained by performing a systematic scan over four dihedral angles describing the orientation of the amino acid group, and the AM1 semiempirical method. The fifth dihedral angle, associated with the bond between the indole and amino acid groups, is kept at $90^{\circ}$ and $270^{\circ}$, respectively. All calculations are performed using the GAUSSIAN 98 package [22]

The structures obtained in the calculations can be classified by their various intramolecular hydrogen bonds. Three main types of hydrogen bonding between the acid and amino groups are possible. First, the hydroxyl hydrogen can hydrogen bond to the amino-nitrogen atom. This structure, in which some added stabilization is found in interactions between the amino hydrogens and the $\pi$ electrons of the indole group, is calculated to have the lowest energy. Second, the aminohydrogen atoms can hydrogen bond to the hydroxyl $\mathrm{O}-\mathrm{H}$ oxygen atom or, in the third case, they can hydrogen bond to the carboxylic $\mathrm{C}=\mathrm{O}$ oxygen atom. Similar results have been obtained by others [14]. 


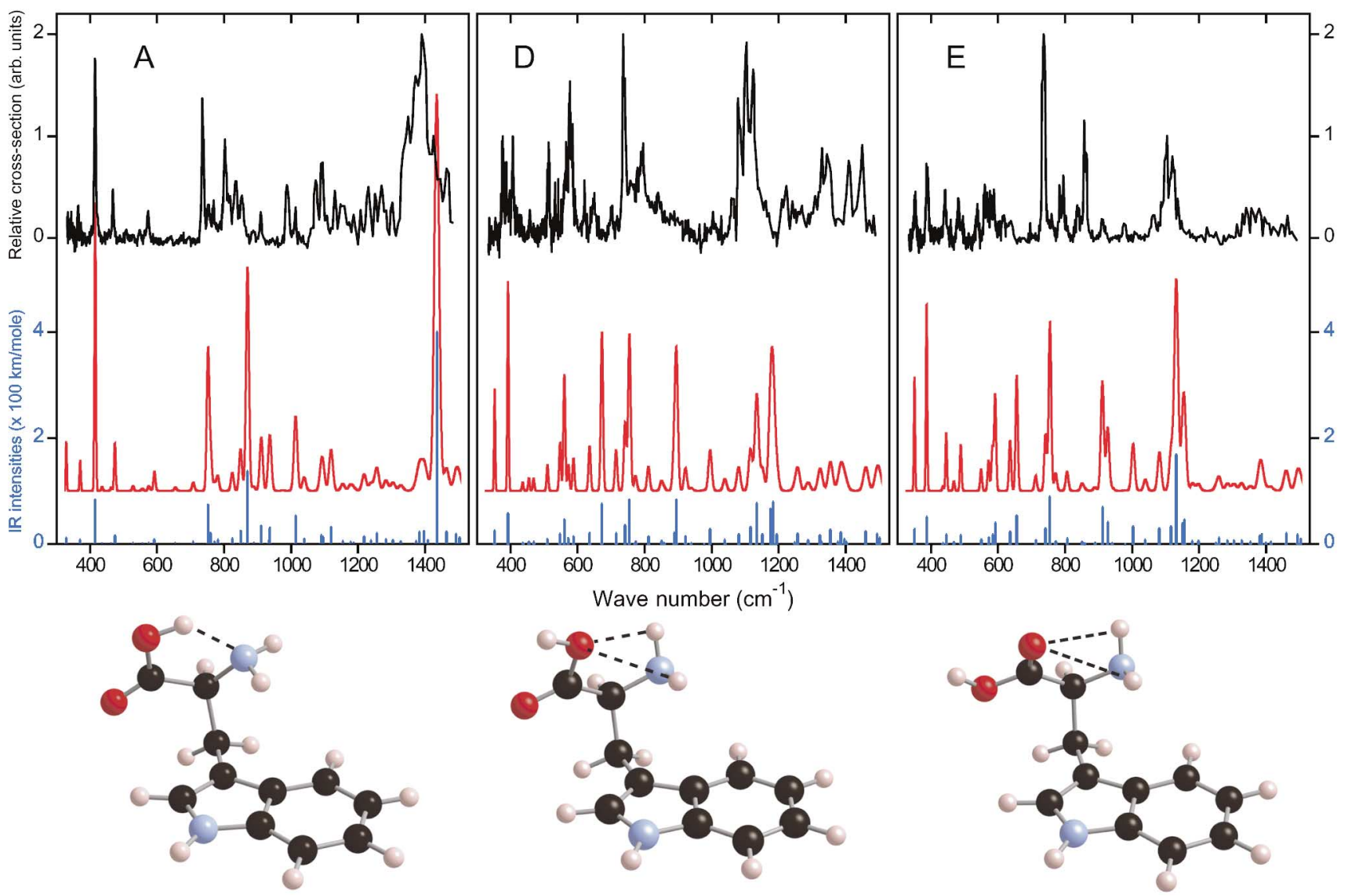

FIG. 2 (color). Infrared absorption spectra of three conformers of tryptophan. Below each experimental spectrum (black), two theoretical spectra are displayed: a stick spectrum (blue) and a convolution of the stick spectrum with the spectral profile of FELIX, which is Gaussian with a bandwidth that is linearly increasing with wave number. The corresponding geometries are displayed below and can be characterized by the orientation of the amino acid group with respect to the indole group. The internal hydrogen bonds that stabilize these orientations are indicated by dashed lines.

For 32 low-energy geometries, the IR absorption spectra are calculated in the harmonic approximation. In Fig. 2, the IR spectra of the three low-energy conformers that show the best match with the experiment are presented as stick spectra and as simulated spectra where the theoretical IR intensities are convoluted with the (frequency dependent) spectral profile of FELIX. No scaling of the calculated frequencies is performed.

The experimental spectrum of conformer A (top trace) matches best with the calculated spectrum of the lowest energy conformer, in which a hydrogen bond between the hydroxyl hydrogen and the amino nitrogen is present. Computer animation of the calculated normal modes reveals that the strongest mode in the spectrum, at $1434 \mathrm{~cm}^{-1}$ is the carboxylic $\mathrm{C}-\mathrm{O}-\mathrm{H}$ bend which is perturbed by the hydrogen bond. Other unique features are the four lowest energy modes below $600 \mathrm{~cm}^{-1}$, of which the strongest, around $400 \mathrm{~cm}^{-1}$, results from the (very much local-mode) out-of-plane motion of the $\mathrm{N}-\mathrm{H}$ in the indole group. The strong resonance at $738 \mathrm{~cm}^{-1}$, which is observed for all conformers close to this frequency, is associated with the indole "umbrella mode." The largest difference between theoretical and experimental spectra is the predicted strength of a fundamental mode at $869 \mathrm{~cm}^{-1}$. The motion associated with this calculated normal mode is essentially an inversion of the amino hydrogens through the (amino acid group) $\mathrm{N}-\mathrm{C}-\mathrm{H}$ plane. This mode is predicted around $900 \mathrm{~cm}^{-1}$ for all calculated structures, but is experimentally not found for any of the three conformers investigated. From earlier experiments, for instance on aniline, it is known, however, that such an inversion mode is rather poorly described in the harmonic approximation [23].

The strong mode around $1400 \mathrm{~cm}^{-1}$, observed for conformer $\mathrm{A}$ and assigned to result from hindered $\mathrm{C}-\mathrm{O}-\mathrm{H}$ bending, is not observed in the spectra of conformers $\mathrm{D}$ and $\mathrm{E}$. This suggests that the $\mathrm{C}-\mathrm{O}-\mathrm{H}$ groups in these two conformers are not involved in hydrogen bonding. IR active modes that involve bending of the $\mathrm{C}-\mathrm{O}-\mathrm{H}$ group are calculated for those conformers as well, but the intensities are predicted to be substantially lower.

A definite assignment of conformer D (middle trace) is more difficult than for conformer A. The resulting 
assignment is based on a good overall agreement between the experimental and the calculated spectra shown in the figure. A key feature in this calculated geometry is the presence of the hydrogen bonds between the amino hydrogens and the hydroxyl oxygen. In addition, the geometry shown is the lowest energy structure with this type of hydrogen bonding.

The spectrum of conformer E (bottom trace) allows for a more solid assignment. The experimental spectrum is observed to have four sharp resonances in the spectral range between 300 and $500 \mathrm{~cm}^{-1}$. These modes are extremely well reproduced in the calculated spectrum. The three lowest-frequency modes represent the indole $\mathrm{N}-\mathrm{H}$ out-of-plane bending vibration, but in this structure they are rather strongly coupled to concerted deformations of the amino acid group. This coupling leads to a distribution of IR intensity over all three modes, which explains the lower intensity relative to that of the localized $\mathrm{N}-\mathrm{H}$ outof-plane bending vibration in conformer A. In the proposed structure for conformer E, hydrogen bonds are present between the amino hydrogens and the carbonyl $\mathrm{C}=\mathrm{O}$ oxygen. A unique feature in the experimental and calculated spectra, which is not found in the spectra of the other calculated structures, is a resonance at $480 \mathrm{~cm}^{-1}$.

The calculated structures that are here assigned to conformers A, D, and E are the lowest energy structures for each of the above-mentioned types of hydrogen bonding. For $\mathrm{A}$ and $\mathrm{E}$, this assignment is the same as that obtained previously from IR experiments in the hydrogen stretching region [14]. For D, a slightly different structure, having the same type of hydrogen bonding, is found to be lower in energy. The differences in energy, structure, and IR spectrum between the conformer D proposed here and the one proposed previously [14] are small, however. Since neither of the two spectra calculated for D agree very well with the experiment, we cannot exclude that this conformer has another not yet considered structure.

The data presented here constitute the first IR measurement of a gas phase biomolecule in the molecular fingerprint region. The combination of a widely tunable IR laser with the ion-dip technique makes it possible to obtain IR spectra that are both mass and conformer specific over a broad spectral range, thereby dramatically increasing the number of vibrations that can be probed. The technique can be extended to larger species as well as towards longer wavelength. However, when the molecules become very large, difficulties arise in bringing them into the gas phase, as well as in selectively ionizing them. For large species, complementary IR spectral information could be obtained from experiments performed on ionic species in the gas phase, generated by MALDI or ESI. In such experiments, a mass change after resonant multiphoton dissociation could be monitored using mass spec- trometry or an IR induced conformational change could be detected by ion mobility measurements.

This work is part of the research program of "Stichting voor Fundamenteel Onderzoek der Materie (FOM)," and it is in part financially supported by the Council for Chemical Sciences (CW), both of which are financially supported by the "Nederlandse Organisatie voor Wetenschappelijk Onderzoek" (NWO).

*Electronic address: jmbakker@rijnh.nl

[1] F. Hillenkamp, M. Karas, R. Beavis, and B. Chait, Anal. Chem. 63, A1193 (1991).

[2] J. Fenn, M. Mann, C. Meng, S. Wong, and C. Whitehouse, Science 246, 64 (1989).

[3] G. von Helden, T. Wyttenbach, and M. T. Bowers, Science 267, 1483 (1995).

[4] D. E. Clemmer, R. Hudgins, and M. F. Jarrold, J. Am. Chem. Soc. 117, 10141 (1995).

[5] D.W. Pratt, Annu. Rev. Phys. Chem. 49, 481 (1998).

[6] T. S. Zwier, Annu. Rev. Phys. Chem. 47, 205 (1996).

[7] T. Ebata, A. Fujii, and N. Mikami, Int. Rev. Phys. Chem. 17, 331 (1998).

[8] F. Dong and R. E. Miller, Science 298, 1227 (2002).

[9] B. C. Dian, A. Longarte, and T. S. Zwier, Science 296, 2369 (2002).

[10] R. H. Page, Y. Shen, and Y. T. Lee, J. Chem. Phys. 88, 4621 (1988).

[11] E. Nir, C. Janzen, P. Imhof, K. Kleinermanns, and M. S. de Vries, J. Chem. Phys. 115, 4604 (2001).

[12] E. Nir, C. Janzen, P. Imhof, K. Kleinermanns, and M. S. de Vries, Phys. Chem. Chem. Phys. 4, 732 (2002).

[13] L. C. Snoek, E. G. Robertson, R. T. Kroemer, and J. P. Simons, Chem. Phys. Lett. 321, 49 (2000).

[14] L. C. Snoek, R. T. Kroemer, M. Hockridge, and J. P. Simons, Phys. Chem. Chem. Phys. 3, 1819 (2001).

[15] M. Gerhards, C. Unterberg, and A. Gerlach, Phys. Chem. Chem. Phys. 4, 5563 (2002).

[16] C. Unterberg, A. Gerlach, T. Schrader, and M. Gerhards, J. Chem. Phys. 118, 8296 (2003).

[17] J. M. Beechem and L. Brand, Annu. Rev. Biochem. 54, 43 (1985).

[18] T. R. Rizzo, Y. D. Park, L. A. Peteanu, and D. H. Levy, J. Chem. Phys. 84, 2534 (1986).

[19] W. B. Colson, E. D. Johnson, M J. Kelley, and H. A. Schwettman, Phys. Today 55, 35 (2002).

[20] D. Oepts, A. F. G. van der Meer, and P. van Amersfoort, Infrared Phys. Technol. 36, 297 (1995).

[21] G. von Helden, D. van Heijnsbergen, and G. Meijer, J. Phys. Chem. A 107, 1671 (2003).

[22] M. J. Frisch et al., GAUSSIAN 98, Revision A.7, Pittsburgh, PA, 1998.

[23] H. Piest, G. von Helden, and G. Meijer, J. Chem. Phys. 110, 2010 (1999). 